\title{
Original
}

\section{Bone Regeneration by Low-dose Recombinant Human Bone Morphogenetic Protein-2 Carried on Octacalcium Phosphate Collagen Composite}

\author{
Nguyen Dien Bien ${ }^{1)}$, Kei-ichiro Miura ${ }^{1)}$, Yoshinori Sumita ${ }^{2)}$, Yuya Nakatani ${ }^{1)}$, Rena Shido ${ }^{1)}$, \\ Fumihiko Kajii $^{3)}$, Shinji Kamakura) and Izumi Asahina ${ }^{1)}$ \\ 1) Department of Regenerative Oral Surgery, Nagasaki University Graduate School of Biomedical Sciences, Nagasaki, Japan \\ 2) Basic and Translational Research Center for Hard Tissue Disease, Nagasaki University Graduate School of Biomedical Sciences, Nagasaki, Japan \\ 3) TOYOBO Co., Ltd., Medical Equipment \& Devices Production Center, Otsu, Japan \\ 4) Division of Bone Regenerative Engineering, Tohoku University Graduate School of Biomedical Engineering, Sendai, Japan \\ (Accepted for publication, March 13, 2020)
}

\begin{abstract}
Bone morphogenetic protein-2 (BMP-2) has diverse functions and is especially important in bone and cartilage development. Recombinant human BMP-2 (rhBMP-2) is an osteoinductive growth factor that has been clinically applied as a bone graft substitute. However, high-dose rhBMP-2 can cause complications such as induction of significant swelling that can endanger the patient's life. Atelocollagen sponge (ACS) is the commercially provided standard carrier of rhBMP-2 in clinical applications. However, a large concentration of rhBMP-2 is required to be clinically effective with ACS as the carrier. Octacalcium phosphate/collagen $(\mathrm{OCP} / \mathrm{Col})$ has been shown to be an excellent bone substitute compared with other bone substitute materials such as hydroxyapatite or $\beta$-tricalcium phosphate due to its biological properties. In this study, we evaluated the use of $\mathrm{OCP} / \mathrm{Col}$ as a carrier to minimize the effective dose of rhBMP-2. ACS or OCP/Col discs impregnated with different rhBMP-2 concentrations were implanted in mice calvarial bone defects. Morphological analysis with micro-CT both at 4 and 6 weeks post-implantation showed homogenous hard tissue formation in the defects of the OCP/Col group at all rhBMP-2 concentrations tested $(0,0.25,0.50$, or $1.00 \mu \mathrm{g})$. In contrast, ACS alone or with $0.25 \mu \mathrm{g}$ of rhBMP-2 showed almost no bone formation. However, bone mineral density in all groups of ACS and OCP/Col was not dependent on rhBMP-2 concentration. Histological evaluation indicated that bone formation progressed depending on rhBMP-2 concentration in the defects of both the ACS and $\mathrm{OCP} / \mathrm{Col}$ groups, although the newly formed bone area was significantly higher in the $\mathrm{OCP} / \mathrm{Col}$ group than in the ACS group. These results indicate that $\mathrm{OCP} / \mathrm{Col}$ could be an effective carrier of rhBMP-2, minimizing the application dose of rhBMP-2 in clinical settings and avoiding the complications caused by high-dose rhBMP-2.
\end{abstract}

Key words: Bone regeneration, Carrier, Collagen, Octacalcium phosphate, Bone morphogenetic protein-2

\section{Introduction}

Bone regeneration is challenging in oral and maxillofacial surgery because there are many cases that require bone regeneration in this region. In current clinical settings, autologous bone grafting remains the gold standard for bone regeneration of jawbone defects because of its superior osteoinductivity and osteoconductivity. However, this technique has several disadvantages such as limited availability and donor site morbidity. Hydroxyapatite (HA) and $\beta$-tricalcium phosphate $(\beta-\mathrm{TCP})$ have been widely applied in clinical settings ${ }^{1-3)}$; however, these materials have not replaced autogenous bone grafts in light of their superior biocompatibility and osteoconductivity but not osteoinductivity ${ }^{4)}$. The clinical application of recombinant human bone morphogenetic protein-2 (rhBMP-2), which is one of the most important bone inductive proteins, has been expected, and it has been approved for clinical use in extraction socket preservation and maxillary sinus floor augmentation ${ }^{5}$. rhBMP-2 shows superior bone regeneration, however, several adverse

Correspondence to: Dr. Kei-ichiro Miura, Department of Regenerative Oral Surgery, Nagasaki University Graduate School of Biomedical Sciences, Japan, 1-7-1, Sakamoto, Nagasaki 852-8588, Japan; Phone: +810958197704; E-mail: kei.miura.e.mail@gmail.com events have been reported such as local edema, seroma, and cancer induction at high-dose rhBMP- ${ }^{6-8)}$. Atelocollagen sponge (ACS) is presently used as a carrier of rhBMP-2; however, it is reported to release rhBMP-2 instantaneously ${ }^{9}$. Accordingly, it is necessary to identify a carrier that will spontaneously release rhBMP-2 and can act as a bone substitute ${ }^{10)}$.

Octacalcium phosphate (OCP) is a direct precursor of biological apatite, which sustainably and irreversibly converts into biological apatite under physiological conditions ${ }^{11}$. Moreover, OCP has been demonstrated to enhance osteoblastic cell differentiation ${ }^{12)}$ and is effective for bone regeneration because of its high bone regenerative ability and rapid absorbability compared with $\mathrm{HA}$ or $\beta-\mathrm{TCP}^{13)}$. OCP possesses many desirable properties as a bone substitute; however, it cannot be molded using sintering processes because of its crystal structure. In order to improve its handling property, a composite comprising OCP and collagen (OCP/ $\mathrm{Col})$ was developed. OCP/Col was shown to yield significantly enhanced bone regeneration compared with $\beta$-TCP or HA collagen composite ${ }^{14)}$. OCP/Col has also been shown to be both safe and efficacious in human tooth extraction sockets, cystic cavities, and maxillary sinus floor elevation ${ }^{15-18)}$. BMP-2 is known to absorb calcium phosphate and 
also collagen ${ }^{19,20)}$. Therefore, we propose that $\mathrm{OCP} / \mathrm{Col}$ could be a superior carrier for rhBMP-2 to minimize the required rhBMP-2 dose. The objective of the present study is to assess the osteogenic potential of low-dose rhBMP-2 carried on OCP/Col compared with that carried on ACS.

\section{Materials and Methods}

Preparation of $\mathrm{OCP}, \mathrm{OCP} / \mathrm{Col}$, atelocollagen, and their combination with/without rhBMP-2

OCP was synthesized by direct precipitation as previously described $^{21)}$, and sieved granules (particle size: $300-500 \mu \mathrm{m}$ ) were obtained. Collagen solution was prepared from NMP collagen PS (Nippon Meat Packers, Tsukuba, Ibaraki, Japan), a lyophilized powder of pepsin-digested atelocollagen isolated from porcine dermis. Collagen was dissolved in distilled water and adjusted to $3 \%$ of the final concentration at $\mathrm{pH}$ 7.4. Then, sieved OCP granules were added to the collagen solution and mixed. The proportion of $\mathrm{OCP}$ in $\mathrm{OCP} / \mathrm{Col}$ was adjusted to 77 weight $\%$. This mixture was then lyophilized and molded into discs (diameter: $9 \mathrm{~mm}$, thickness: $1 \mathrm{~mm}$ ). OCP discs then underwent dehydrothermal treatment $\left(150^{\circ} \mathrm{C}, 24 \mathrm{~h}\right)$ in a vacuum drying oven and sterilization using gamma-ray irradiation ( $5 \mathrm{kGy}$ ). The resultant discs were punched to $5 \mathrm{~mm}$ in diameter using a HANDY PUNCH KIT (product number: 3H-PS10, H.H.H. Manufacturing Co., Osaka, Japan). rhBMP-2 (produced by INFUSE ${ }^{\circledR}$ Bone Graft, Medtronic, Memphis, TN, USA) was dissolved in distilled water at concentrations of $0,0.01,0.02$, or $0.04 \mu \mathrm{g} / \mu \mathrm{l}$. Then, $25 \mu \mathrm{l}$ of rhBMP-2 solution was dripped onto the $\mathrm{OCP} /$ Col or ACS disc (control group; diameter: $5 \mathrm{~mm}$, thickness: $1 \mathrm{~mm}$ ). These materials were defined as $\mathrm{OCP} / \mathrm{Col}, 0.25 \mathrm{OCP} / \mathrm{Col}, 0.50 \mathrm{OCP} /$ Col, $1.00 \mathrm{OCP} / \mathrm{Col}$, ACS, 0.25 ACS, 0.50 ACS, and 1.00 ACS.

\section{Animals and implantation procedures}

All animal experiments were performed at the Nagasaki University Animal Experiment Facility and were in accordance with protocols approved by the Local Institutional Animal Care and Use Committee of Nagasaki University (approval no. 1606211320). Eighty 10-week-old male C57BL/6J mice (CLEA Japan, Inc., Tokyo, Japan) were used. Mice were housed in standard rodent cages in a light- and temperature-controlled room in the Biomedical Research Center, Center for Frontier Life Sciences, Nagasaki University. Animals had free access to standard laboratory chow and tap water. Transplantation experiments were conducted under general anesthesia. General anesthetics were prepared by mixing $1.875 \mathrm{ml}$ of medetomidine hydrochloride $(1 \mathrm{mg} / \mathrm{ml}), 2$ $\mathrm{ml}$ of midazolam $(5 \mathrm{mg} / \mathrm{ml}), 2.5 \mathrm{ml}$ of butorphanol $(5 \mathrm{mg} / \mathrm{ml})$, and $18.625 \mathrm{ml}$ of physiological saline to yield a final liquid volume of 25 $\mathrm{ml}$. The mixture was then subcutaneously or intraperitoneally injected at a concentration of $0.1 \mathrm{ml} / 10 \mathrm{~g}$ body weight. After disinfection of the operative field, an arc-shaped skin incision was made from the left to right preauricular region via the forehead region. The periosteum of the calvarium was ablated and a full-thickness standardized trephine defect, 5 $\mathrm{mm}$ in diameter, was made in the calvarium under continuous saline buffer irrigation. Each material was then implanted into the trephine defect. Five mice were treated per group. After the defects were treated, the ablated periosteum and skin were repositioned and sutured. For the $\mathrm{OCP} / \mathrm{Col}$ and ACS groups, the tissues were collected at four and six weeks postoperatively.

\section{Morphological and quantitative analysis by micro-computed tomogra- phy}

The morphological and quantitative image analysis of newly formed bone was performed using micro-computed tomography (CT) [micro X-ray CT machine for laboratory animals (R mCT), RIGAKU, Tokyo, Japan] under standardized conditions ( $90 \mathrm{kV}, 150 \mathrm{~mA}$, two minutes). In three-dimensional analysis using structural analysis software (TRI/3DBON, RATOC System Engineering, Osaka, Japan), the newly formed bone area was analyzed after sampling using bone mineral density (BMD) standards, of established densities of between $300 \mathrm{mg} / \mathrm{cm}^{3}$ and $1,500 \mathrm{mg} / \mathrm{cm}^{3}$, and the extracted range of bone was defined between 300 $\mathrm{mg} / \mathrm{cm}^{3}$ and $1,100 \mathrm{mg} / \mathrm{cm}^{3}$.

\section{Tissue preparation and histological examination}

After micro-CT examination, samples were immersed in PBS overnight and fixed in 4\% paraformaldehyde for $24 \mathrm{~h}$. Samples were then decalcified in EDTA for 10 days and washed with distilled water. Thereafter, samples were cut coronally into two pieces at the center of the defect and embedded in paraffin. Serial sections $5 \mu \mathrm{m}$ thick were cut coronally and stained with hematoxylin and eosin. Images were taken using a photomicroscope (Axiocam ERc 5s, ZEISS, Oberkochen, Germany).

\section{Quantitative measurement of cortical bone and bone marrow area}

The amount of cortical bone and bone marrow area in the implanted material was measured from the histological sections prepared near the center of the defect. The cortical bone and bone marrow data was extracted using Adobe Photoshop ${ }^{\circledR}$ CS6 Extended. After converting to JPEG images, the data volume (Kilobytes) of cortical bone and bone marrow was measured with Windows 10 photo viewer.

\section{Statistical analysis}

Statistical analysis of all micro-CT data was performed using JMP ${ }^{\circledR}$ version 13 (SAS Institute, Cary, NC, USA). The temporal change of each $\mathrm{OCP} / \mathrm{Col}$ group's $\mathrm{BMD}$ value was analyzed by a nonparametric multiple comparison test. Values are expressed as mean \pm standard deviation (SD). A p-value less than 0.05 was statistically significant.

\section{Morphological analysis by micro-CT}

\section{Results}

Micro-CT analysis revealed that the ACS and $0.25 \mathrm{ACS}$ groups showed almost no hard tissue formation, whereas the 0.50 ACS and 1.00 ACS groups showed hard tissue formation. The formation area was slightly wider in the 1.00 ACS group at both 4 and 6 weeks postoperatively compared with the 0.50 ACS group. In contrast, hard tissue formation was recognized within the defect in the $\mathrm{OCP} / \mathrm{Col}$ groups at all rhBMP-2 concentrations, and the radio-lucent area was decreased rhBMP-2 dose-dependently (Fig. 1).

\section{Morphometric analysis of newly formed bone volume by micro-CT analysis}

The OCP/Col groups produced a greater volume of newly formed bone than the ACS groups. Namely, $0.25 \mathrm{OCP} / \mathrm{Col}, 0.50 \mathrm{OCP} / \mathrm{Col}$, and $1.00 \mathrm{OCP} / \mathrm{Col}$ showed significant increases in newly formed bone volume compared with 1.00 ACS, which showed the highest bone volume among the ACS groups, at four weeks, while $1.00 \mathrm{OCP} / \mathrm{Col}$ showed significant increases in bone volume compared with 1.00 ACS at 6 weeks (Fig. 2).

\section{Bone mineral density (BMD)}

BMD tended to increase from 4 to 6 weeks postimplantation in all groups. There were also no significant differences in BMD between the ACS and OCP/Col groups. For example, BMD of $1.00 \mathrm{OCP} / \mathrm{Col}$, which 
Nguyen Dien Bien et al:: Bone Regeneration by Low-dose rhBMP-2 Carried on OCP/Col Composite

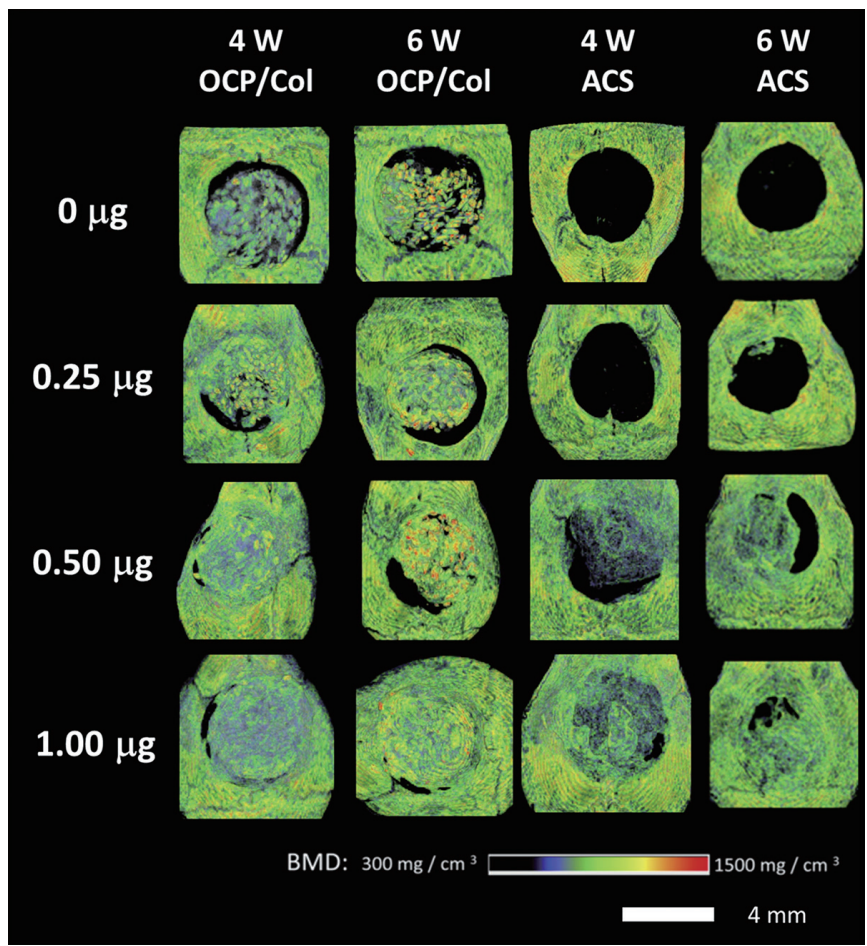

Figure 1. Tissue formation in the ACS and OCP/Col groups with and without rhBMP-2 at 4 and 6 weeks postoperatively. The ACS and 0.25 ACS groups showed almost no hard tissue formation, whereas the $0.50 \mathrm{ACS}$ and 1.00 ACS groups showed hard tissue formation. The formation area was slightly wide in the $1.00 \mathrm{ACS}$ group at both 4 and 6 weeks postoperatively. In contrast, uniform hard tissue formation was recognized within the defect in the $\mathrm{OCP} / \mathrm{Col}$ groups at all rhBMP-2 concentrations. produced the highest bone volume, was not significant different compared with ACS alone both at four and six weeks (Fig. 3).

\section{Histological evaluation}

ACS and 0.25 ACS showed no bone formation in the defect, whereas 0.50 and $1.00 \mathrm{ACS}$ showed a certain amount of new bone formation at four weeks. On the other hand, in the $\mathrm{OCP} / \mathrm{Col}$ groups, the area of new bone gradually increased as the concentration of rhBMP-2 increased (Fig. 4). ACS and 0.25 ACS showed slight bone formation in the defect, and 1.00 ACS demonstrated bone bridging of the defect with new bone at six weeks. In contrast, the OCP/Col group showed remarkable osteogenesis compared with the ACS group (Fig. 5). Almost all implanted ACS was absorbed in all ACS groups, while $\mathrm{OCP} / \mathrm{Col}$ remained, especially at the lower concentrations of rhBMP-2. Additionally, newly formed bone in the $\mathrm{OCP} / \mathrm{Col}$ groups was thicker than that in the ACS groups. Although OCP/Col without rhBMP-2 showed new bone formation, OCP/Col with rhBMP-2 showed more mature bone formation with bone marrow.

\section{Quantitative measurement of cortical bone and bone marrow area}

The area of newly formed cortical bone and bone marrow area measured using histological specimens showed a similar tendency, that is, bone volume measured with micro-CT increased in a rhBMP-2 dose-dependent manner for both the ACS and $\mathrm{OCP} / \mathrm{Col}$ groups. The area of the $\mathrm{OCP} / \mathrm{Col}$ groups was higher than that of the ACS groups at the respective concentrations of rhBMP-2. Statistically significant differences were observed between the lower $(0,0.25)$ and higher $(0.50,1.00)$ concentrations of rhBMP-2 in both the ACS and OCP/Col groups (Fig. 6). $0.25 \mathrm{OCP} / \mathrm{Col}$, which was the lowest concentration of rhBMP-2, showed similar bone area with 0.50 and 1.00 ACS at 6 weeks.

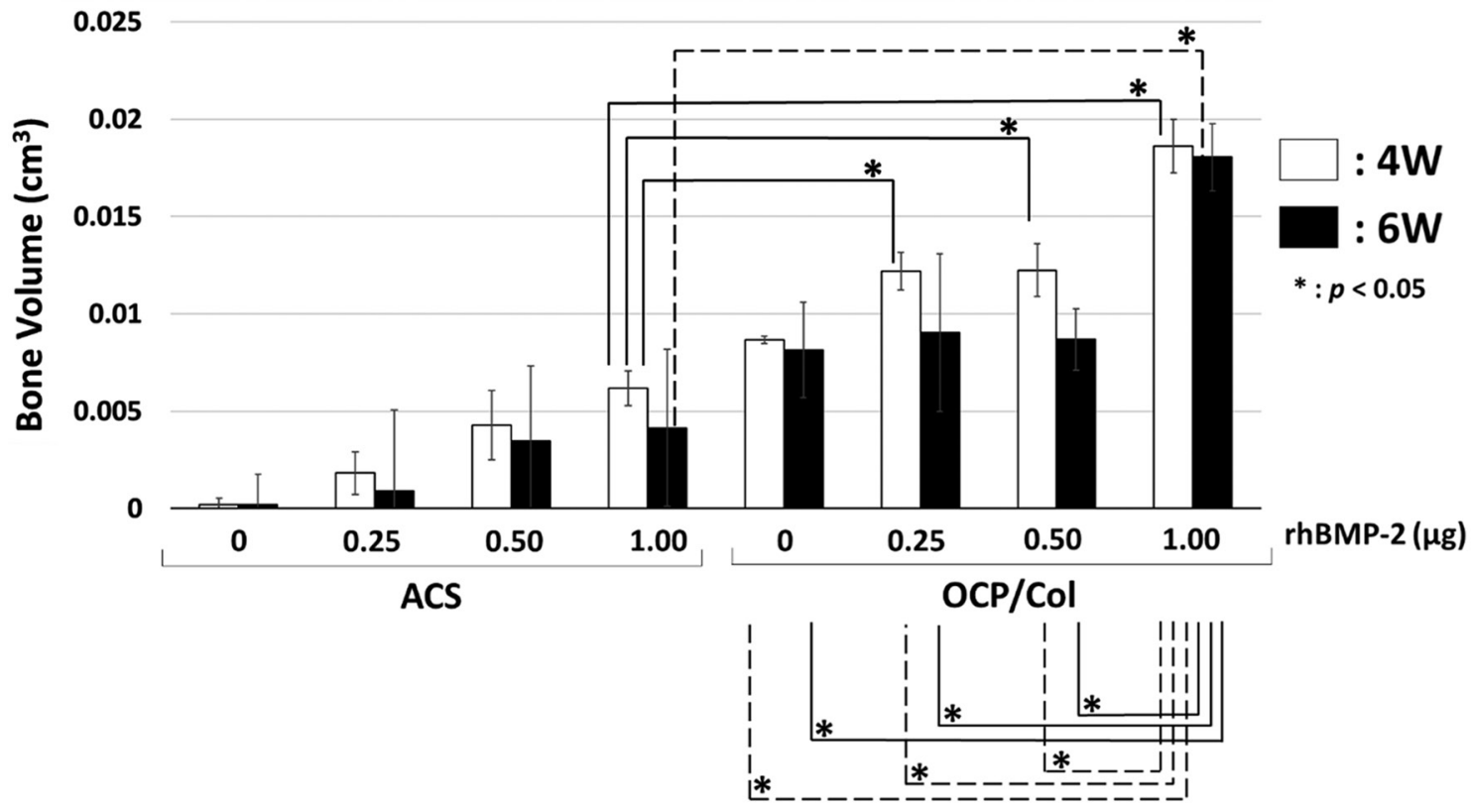

Figure 2. Bone volume in the ACS and OCP/Col groups with and without rhBMP-2 at 4 and 6 weeks postoperatively. At 4 weeks, 0.25 OCP/Col, $0.50 \mathrm{OCP} / \mathrm{Col}$, and $1.00 \mathrm{OCP} / \mathrm{Col}$ showed significant increases in newly formed bone volume compared with $1.00 \mathrm{ACS}$. At 6 weeks, $1.00 \mathrm{OCP} /$ Col showed significant increases in bone volume compared with $1.00 \mathrm{ACS}$, whereas, OCP/Col, $0.25 \mathrm{OCP} / \mathrm{Col}$, and $0.50 \mathrm{OCP} / \mathrm{Col} \mathrm{did}$ not. * : $\mathrm{p}<0.05$ 
n.s.

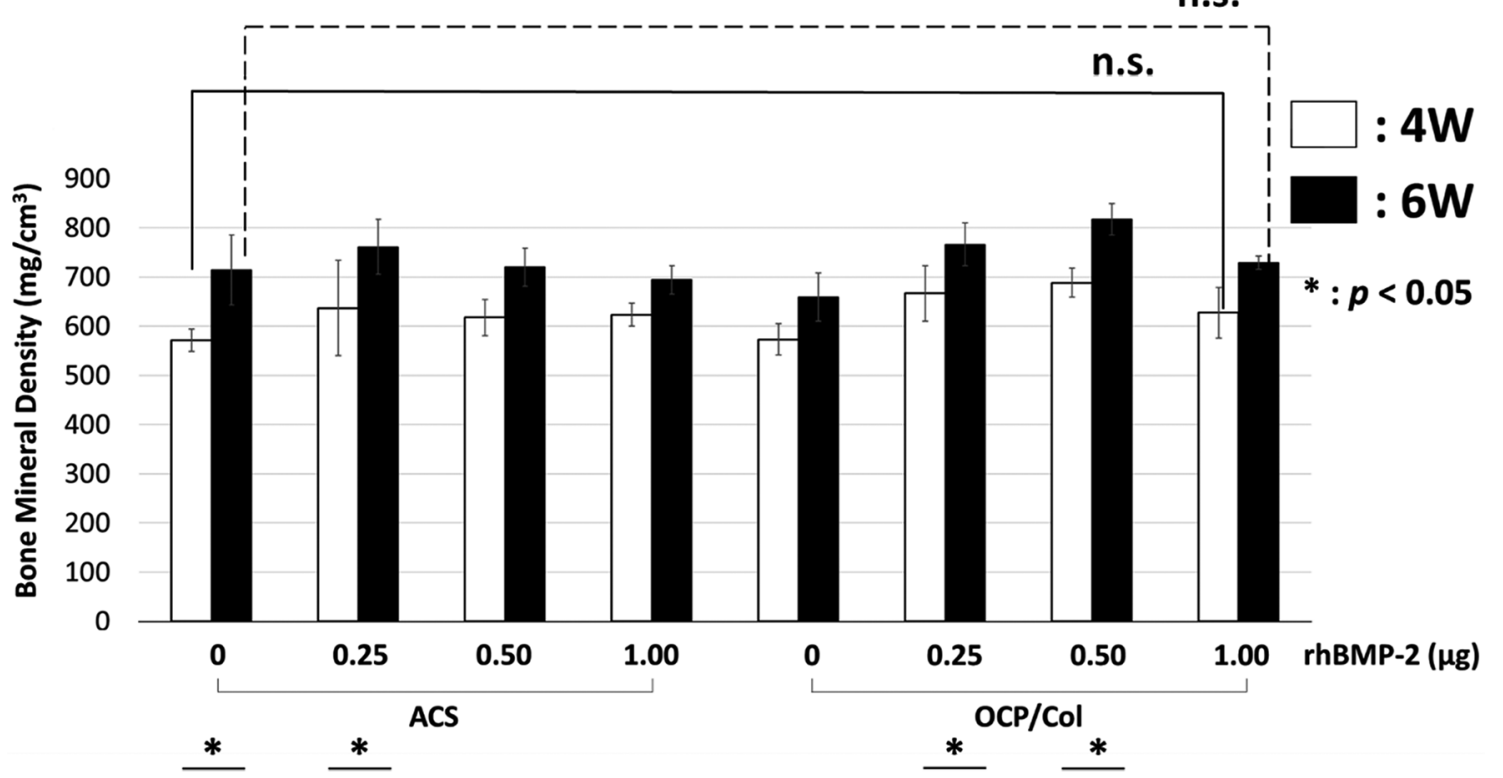

Figure 3. Bone mineral density in the ACS and OCP/Col groups with and without rhBMP-2. At both 4 and 6 weeks postoperatively, the bone mineral density of $1.00 \mathrm{OCP} / \mathrm{Col}$ with rhBMP-2 was not significantly different compared with ACS without rhBMP-2. * $: \mathrm{p}<0.05$

4W ACS/rhBMP-2

$0 \mu g$

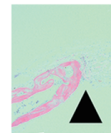

$0.25 \mu \mathrm{g}$

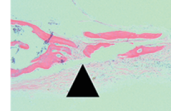

$0.50 \mu \mathrm{g}$

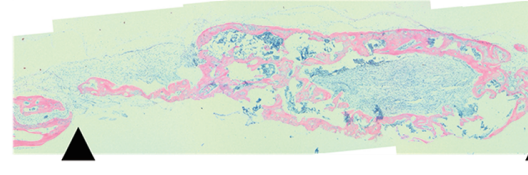

$\Delta$

a

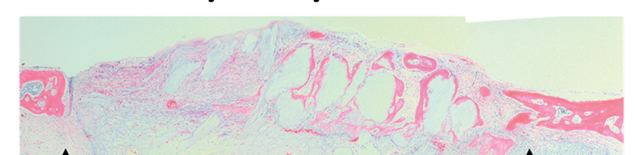

$\Delta$

$\Delta$

e

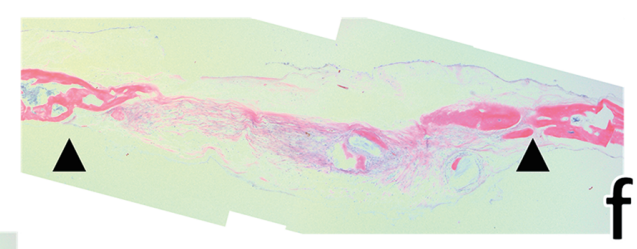

b
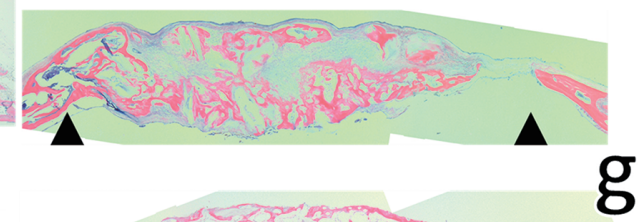

C

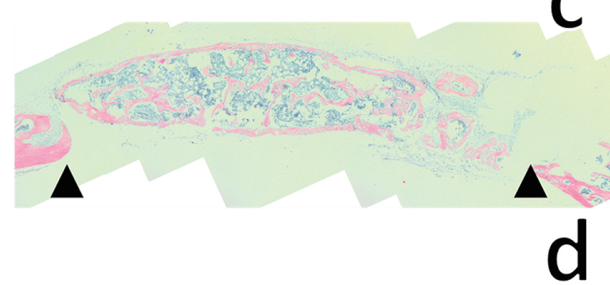

g

$1.00 \mu \mathrm{g}$

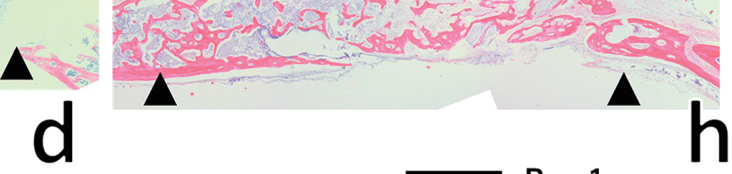

Bar: $1 \mathrm{~mm}$

Figure 4. Effect of ACS and OCP/Col with and without rhBMP-2 on bone formation in defects at 4 weeks postoperatively. At 4 weeks ACS and $0.25 \mathrm{ACS}$ showed no bone formation in the defect, whereas 0.50 and $1.00 \mathrm{ACS}$ showed slight new bone formation. On the other hand, in the $\mathrm{OCP} / \mathrm{Col}$ groups, the area of new bone gradually increased as the concentration of rhBMP-2 increased. 
$6 W \quad$ ACS/rhBMP-2 OCP/Col/rhBMP-2

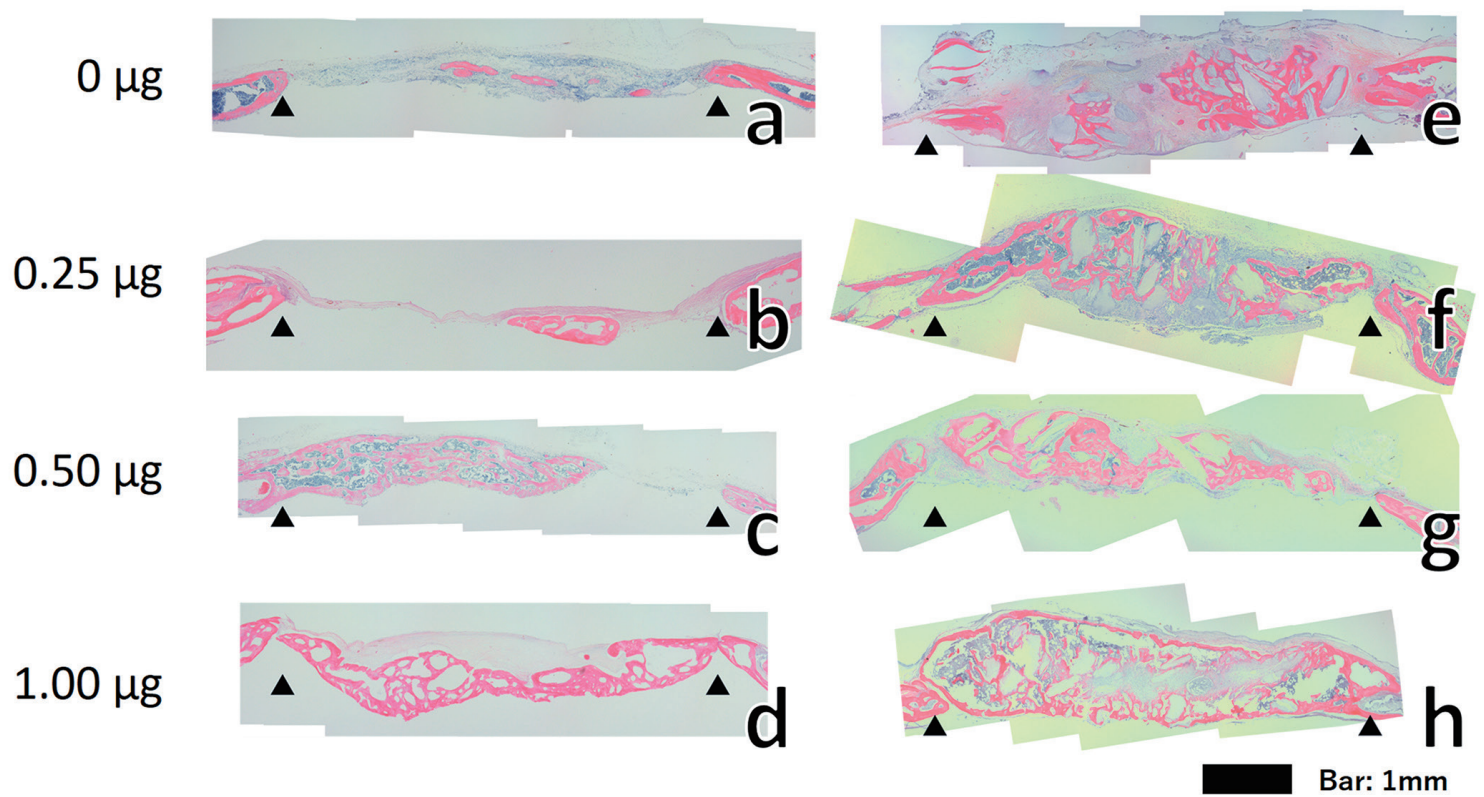

Figure 5. Effect of ACS and $\mathrm{OCP} / \mathrm{Col}$ with and without rhBMP-2 on bone formation in defects at 6 weeks postoperatively. At 6 weeks the ACS and 0.25 ACS groups showed slight formation in the defect, and the 1.00 ACS group showed bone bridging of the defect with new bone. In contrast, the OCP/Col group showed remarkable osteogenesis compared with the ACS group. Although OCP/Col without rhBMP-2 showed remarkable new bone formation, OCP/Col with rhBMP-2 showed more mature bone formation with bone marrow. Additionally, newly formed bone in the $\mathrm{OCP} / \mathrm{Col}$ groups was thicker than that in the ACS groups.

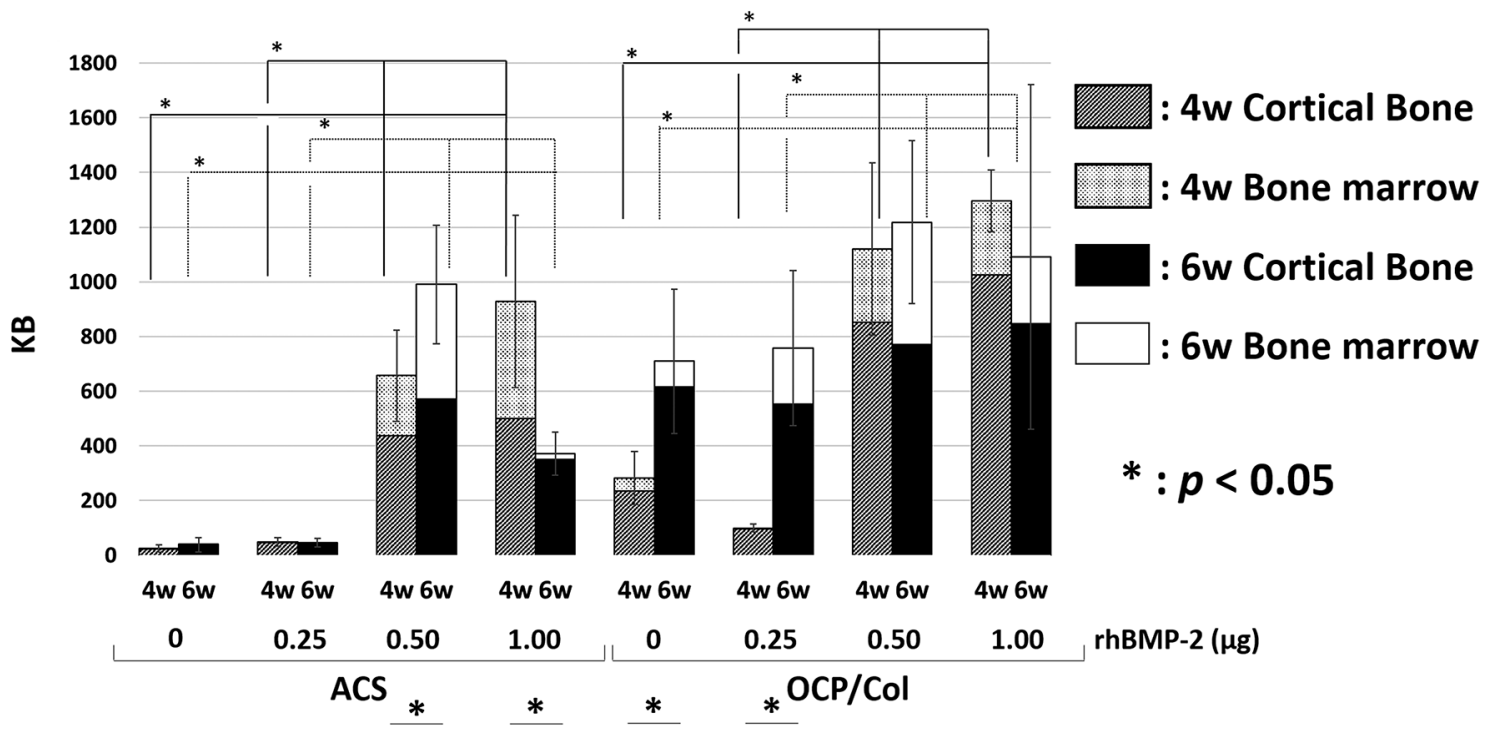

Figure 6. Effect of ACS and OCP/Col with and without rhBMP-2 on areas of cortical bone and bone marrow at 4 and 6 weeks postoperatively. The areas of cortical bone and bone marrow were increased in an rhBMP-2 dose-dependent manner in both the ACS and OCP/Col groups, and the OCP/Col groups showed higher bone areas than the ACS groups. Statistically significant differences were observed between the lower $(0$, $0.25)$ and higher $(0.50,1.00)$ concentrations of rhBMP-2 in both the ACS and OCP/Col groups. ${ }^{*}: \mathrm{p}<0.05$

\section{Discussion}

In the current study, we examined the potential application of OCP/ Col as an rhBMP-2 carrier to minimize the effective dose of rhBMP-2 on bone regeneration. Our findings demonstrate that low-concentration rhBMP-2 impregnated on $\mathrm{OCP} / \mathrm{Col}$ discs could induce sufficient bone formation. According to the qualitative analysis using micro-CT and histological findings, the concentration of rhBMP-2 could be decreased to less than one-fourth using $\mathrm{OCP} / \mathrm{Col}$ as the carrier compared with $\mathrm{ACS}$ (Figs. 2 and 6). In terms of clinical application of BMP, selection of an appropriate carrier is extremely important for the following reasons. Since rhBMP-2 diffuses within a short time when topically administered without a carrier ${ }^{22)}$, an appropriate carrier for proper delivery, retention, 
and sustained released of BMP-2 is needed ${ }^{19)}$. The carrier must be nontoxic, noncarcinogenic, nonantigenic, proinflammatory, and preferably biodegradable and moldable ${ }^{23}$. There are four major categories of rhBMP-2 carriers: natural-origin polymers, inorganic materials, synthetic biodegradable polymers, and composites ${ }^{24)}$. In current clinical settings, ACS is the commercially provided standard carrier for rhBMP-2 among the presently available carriers that fulfill the requirements described above $^{25)}$. However, a high rhBMP-2 concentration is required when ACS is used as the carrier ${ }^{17}$. As previously demonstrated, high doses of BMP2 cause severe clinical side effects such as ectopic bone formation, osteoclast-mediated bone resorption, inappropriate adipogenesis, and cancer $^{19,26)}$. Additionally, high-dose rhBMP-2 can induce acute edema and swelling, leading to fatal airway obstruction in the oral and maxillofacial region ${ }^{6,27}$. Therefore, low-dose rhBMP-2 should be applied to avoid these adverse effects. In the present clinical settings, the concentration of rhBMP-2 with ACS as a carrier is $37.5 \mu \mathrm{g} / \mu \mathrm{l}$. Even taking into an account the differences between humans and mice in the response to BMP, it is expected that the concentration of rhBMP-2 can be reduced when $\mathrm{OCP} / \mathrm{Col}$ is used as a carrier.

There are a number of reasons why $\mathrm{OCP} / \mathrm{Col}$ is a potential superior carrier for rhBMP-2 compared with ACS. First, in accordance with a previous report, $\mathrm{OCP} / \mathrm{Col}$ itself has bone inductive activity ${ }^{28)}$. As shown in Figs. 1, 4 and 5, new bone formation was observed apart from the rim of the bone defect in the OCP/Col only group (without rhBMP-2), indicating that $\mathrm{OCP} / \mathrm{Col}$ shows osteoconductivity as well as osteoinductivity. It is apparent that the bone inductive activity of rhBMP-2 augments that of $\mathrm{OCP} / \mathrm{Col}$, since $\mathrm{OCP} / \mathrm{Col}$ with $\mathrm{rhBMP}-2$ induced more mature bone formation with cortical bone and bone marrow compared with OCP/Col without rhBMP-2 (Figs. 1, 4 and 5). BMD tended to increase time-dependently, but significant differences were not observed. There were also no significant differences among the ACS and $\mathrm{OCP} / \mathrm{Col}$ groups with different concentrations of rhBMP-2 (Fig. 3). This suggests that while the quality of bone is similar, the quantity is different between $\mathrm{ACS}$ and $\mathrm{OCP} / \mathrm{Col}$ as carriers. Second, the composition of $\mathrm{OCP} / \mathrm{Col}$ is favorable for use as an rhBMP-2 carrier because rhBMP-2 is known to bind tightly to calcium phosphate and collagen, which are major components of $\mathrm{OCP} / \mathrm{Col}^{19,20)}$. It is speculated that rhBMP-2 is released as OCP and collagen are degraded, allowing the slow release of rhBMP- $2^{26,27)}$. Remnants of $\mathrm{OCP} / \mathrm{Col}$ were observed at 6 weeks postimplantation, while ACS was completely absorbed. This might assist in the sustainable release of rhBMP-2 with $\mathrm{OCP} / \mathrm{Col}$ as the carrier. Furthermore, rhBMP-2 influences chemotaxis and mesenchymal stem cells proliferation and differentiation into osteoblasts, thus, the porous structure of OCP/ Col, composed of collagen, is a suitable environment for cell migration $^{29)}$. Lastly, OCP/Col has superior mechanical strength to $\mathrm{ACS}^{30}$. We observed that newly formed bone in the $\mathrm{OCP} / \mathrm{Col}$ implant group was thicker than that in the ACS implant groups (Figs. 4 and 5). This finding suggests that $\mathrm{OCP} / \mathrm{Col}$ is sufficiently strong to resist pressure from the skin flap. rhBMP-2/ACS has been approved for clinical application for socket preservation and sinus floor augmentation in the oral and maxillofacial region ${ }^{31)}$. In these areas, the implant material does not suffer from pressure because it is surrounded by the bone wall. However, the implant material needs to have certain mechanical strength in the case of alveolar bone ridge augmentation. In this respect, rhBMP-2 with $\mathrm{OCP} / \mathrm{Col}$ could be a good implant material for guided bone regeneration (GBR) because it possesses both strength and flexibility.

In the present study, we demonstrated superior bone regeneration of rat calvarial bone defects with rhBMP-2 carried on OCP/Col. Although $\mathrm{OCP} / \mathrm{Col}$ itself has bone inductive activity, low-dose rhBMP-2 enhances this activity. The minimization of the effective dose of rhBMP-2 reduces its side effects. Recently, there has been increasing demand for alveolar bone regeneration in response to the popularization of implant dentistry. We suggest that rhBMP-2 with OCP/Col could be an ideal implant material for GBR because of its superior osteoinductivity and mechanical properties such as strength and flexibility. In conclusion, though OCP/ $\mathrm{Col}$ itself is a good bone substitute, it could also be an effective carrier for rhBMP-2, thereby reducing the effective dose of rhBMP-2 and resulting in its safe clinical application for more reliable bone augmentation.

\section{Acknowledgements}

This study was partially supported by Grants-in-Aid for Scientific Research from the Japan Society for the Promotion of Science (No. 17H01604).

\section{Conflict of Interest}

The authors have declared that no COI exists.

\section{References}

1. LeGeros RZ. Properties of osteoconductive biomaterials: calcium phosphates. Clin Orthop Relat Res 395: 81-98, 2002

2. Ogose A, Hotta T, Kawashima H, Kondo N, Gu W, Kamura T and Endo N. Comparison of hydroxyapatite and beta tricalcium phosphate as bone substitutes after excision of bone tumors. J Biomed Mater Res B Appl Biomater 72: 94-101, 2005

3. Ogose A, Kondo N, Umezu H, Hotta T, Kawashima H, Tokunaga K, Ito T, Kudo N, Hoshino M and Gu W. Histological assessment in grafts of highly purified beta-tricalcium phosphate (OSferion $\left.{ }^{\circledR}\right)$ in human bones. Biomaterials 27: 1542-1549, 2006

4. Lichte P, Pape HC, Pufe T, Kobbe P and Fischer H. Scaffolds for bone healing: concepts, materials and evidence. Injury 42: 569-573, 2011

5. Asahina I. Bone morphogenetic proteins: their history and characteristics. J Hard Tissue Biol 23: 283-286, 2014

6. Woo EJ. Adverse events reported after the use of recombinant human bone morphogenetic protein 2. J Oral Maxillofac Surg 70: 765767, 2012

7. Carreira A, Lojudice F, Halcsik E, Navarro R, Sogayar M and Granjeiro J. Bone morphogenetic proteins: facts, challenges, and future perspectives. J Dent Res 93: 335-345, 2014

8. Sugamori Y, Mise-Omata S, Maeda C, Aoki S, Tabata Y, Murali R, Yasuda H, Udagawa N, Suzuki H, Honma M and Aoki K. Peptide drugs accelerate BMP-2-induced calvarial bone regeneration and stimulate osteoblast differentiation through $\mathrm{mTORC} 1$ signaling. Bioessays 38: 717-725, 2016

9. Brown KV, Li B, Guda T, Perrien DS, Guelcher SA and Wenke JC. Improving bone formation in a rat femur segmental defect by controlling bone morphogenetic protein-2 release. Tissue Eng Part A 17: 1735-1746, 2011

10. Kawai T, Kamakura S, Matsui K, Fukuda M, Takano H, Iino M, Ishikawa S, Kawana H, Soma T, Imamura E, Kizu H, Michibata A, Asahina I, Miura K, Nakamura N, Kibe T, Suzuki O and Takahashi T. Clinical study of octacalcium phosphate and collagen composite in oral and maxillofacial surgery. J Tissue Eng 11: 2041731419896449, 2020

11. Brown WE, Smith JP, Lehr JR and Frazier AW. Octacalcium phosphate and hydroxyapatite: crystallographic and chemical relations between octacalcium phosphate and hydroxyapatite. Nature 196: 
$1050-1055,1962$

12. Suzuki O, Kamakura S, Katagiri T, Nakamura M, Zhao B, Honda Y and Kamijo R. Bone formation enhanced by implanted octacalcium phosphate involving conversion into Ca-deficient hydroxyapatite. Biomaterials 27: 2671-2681, 2006

13. Kamakura S, Sasano Y, Shimizu T, Hatori K, Suzuki O, Kagayama $\mathrm{M}$ and Motegi K. Implanted octacalcium phosphate is more resorbable than $\beta$-tricalcium phosphate and hydroxyapatite. J Biomed Mater Res Part A 59: 29-34, 2002

14. Kamakura S, Sasaki K, Homma T, Honda Y, Anada T, Echigo S and Suzuki O. The primacy of octacalcium phosphate collagen composites in bone regeneration. J Biomed Mater Res A 83: 725-733, 2007

15. Kawai T, Echigo S, Matsui K, Tanuma Y, Takahashi T, Suzuki O and Kamakura S. First clinical application of octacalcium phosphate collagen composite in human bone defect. Tissue Eng Part A 20: 1336-1341, 2014

16. Kawai T, Suzuki O, Matsui K, Tanuma Y, Takahashi T and Kamakura S. Octacalcium phosphate collagen composite facilitates bone regeneration of large mandibular bone defect in humans. J Tissue Eng Regen Meddoi: 10.1002/term.2110, 2015

17. Kawai T, Tanuma Y, Matsui K, Suzuki O, Takahashi T and Kamakura S. Clinical safety and efficacy of implantation of octacalcium phosphate collagen composites in tooth extraction sockets and cyst holes. J Tissue Eng 7: 2041731416670770, 2016

18. Miura K-i, Sumita Y, Kajii F, Tanaka H, Kamakura S and Asahina I. First clinical application of octacalcium phosphate collagen composite on bone regeneration in maxillary sinus floor augmentation: A prospective, single-arm, open-label clinical trial. J Biomed Mater Res B Appl Biomater 108: 243-252, 2020

19. Lee YH, Lee BW, Jung YC, Yoon BI, Woo HM and Kang BJ. Application of alginate microbeads as a carrier of bone morphogenetic protein-2 for bone regeneration. J Biomed Mater Res B Appl Biomater 107: 286-294, 2019

20. Kamakura S, Nakajo S, Suzuki O and Sasano Y. New scaffold for recombinant human bone morphogenetic protein-2. J Biomed Mater Res A 71: 299-307, 2004

21. Suzuki O, Nakamura M, Miyasaka Y, Kagayama M and Sakurai M. Bone Formation on Synthetic Precursors of Hydroxyapatite. Tohoku J Exp Med 164: 37-50, 1991
22. Takaoka K, Nakahara H, Yoshikawa H, Masuhara K, Tsuda T and Ono $\mathrm{K}$. Ectopic bone induction on and in porous hydroxyapatite combined with collagen and bone morphogenetic protein. Clinical orthopaedics and related research 234: 250-254, 1988

23. Takahashi K. Bone morphogenetic protein (BMP): from basic studies to clinical approaches. Folia Pharmacologica Japonica 116: 232240, 2000

24. Haidar ZS, Hamdy RC and Tabrizian M. Delivery of recombinant bone morphogenetic proteins for bone regeneration and repair. Part A: Current challenges in BMP delivery. Biotechnol Lett 31: 18171824, 2009

25. Bessa PC, Casal M and Reis R. Bone morphogenetic proteins in tissue engineering: the road from laboratory to clinic, part II (BMP delivery). J Tissue Eng Regen Med 2: 81-96, 2008

26. Boerckel JD, Kolambkar YM, Dupont KM, Uhrig BA, Phelps EA, Stevens HY, García AJ and Guldberg RE. Effects of protein dose and delivery system on BMP-mediated bone regeneration. Biomaterials 32: 5241-5251, 2011

27. Yaremchuk K, Toma M and Somers M. Acute airway obstruction associated with the use of bone-morphogenetic protein in cervical spinal fusion. Laryngoscope 120: S140-S140, 2010

28. Kouketsu A, Matsui K, Kawai T, Ezoe Y, Yanagisawa T, Yasuda A, Takahashi $\mathrm{T}$ and Kamakura S. Octacalcium phosphate collagen composite stimulates the expression and activity of osteogenic factors to promote bone regeneration. J Tissue Eng Regen Med 14: $99-$ 107,2020

29. Neovius E, Lemberger M, Skogh AD, Hilborn J and Engstrand T. Alveolar bone healing accompanied by severe swelling in cleft children treated with bone morphogenetic protein-2 delivered by hydrogel. J Plast Reconstr Aesthet Surg 66: 37-42, 2013

30. Kamakura S, Sasaki K, Honda Y, Anada T and Suzuki O. Octacalcium phosphate combined with collagen orthotopically enhances bone regeneration. J Biomed Mater Res B Appl Biomater 79: 210-217, 2006

31. Triplett RG, Nevins M, Marx RE, Spagnoli DB, Oates TW, Moy PK and Boyne PJ. Pivotal, randomized, parallel evaluation of recombinant human bone morphogenetic protein-2/absorbable collagen sponge and autogenous bone graft for maxillary sinus floor augmentation. J Oral Maxillofac Surg 67: 1947-1960, 2009 
J.Hard Tissue Biology Vol. 29(2): 123-130, 2020 